\title{
Las situaciones de exclusión social como factor de vulnerabilidad a la violencia de género en la pareja: Desigualdades estructurales y relaciones de poder de género
}

\author{
Situations of social exclusion as a vulnerability factor for \\ gender-based violence in intimate relationships: Structural \\ inequalities and gender power relations
}

\author{
Paola Damonti \\ Universidad Pública de Navarra \\ paola.damonti84@gmail.com (ESPAÑA) \\ Patricia Amigot Leache \\ Universidad Pública de Navarra
}

Recibido: 20.02 .2019

Aceptado: 01.06.2020

\section{RESUMEN}

Partiendo de la evidencia de que, en contextos de exclusión social, la prevalencia de violencia de género en la pareja se incrementa, hemos querido analizar las dinámicas de la relación entre estos dos fenómenos. Para ello, realizamos 16 entrevistas en profundidad a mujeres supervivientes, que evidenciaron que la situación de exclusión podía ser tanto un factor desencadenante como un producto de dicha violencia. Aquí analizamos en detalle el primer recorrido e identificamos una serie de elementos que pueden favorecer la aparición de violencia. Los clasificamos en dos grandes grupos: por un lado, factores que condicionan el proceso de formación de pareja; por otro, factores que facilitan la aparición de violencia de género en una pareja ya constituida. Entre los primeros cabe señalar la existencia, en determinadas situaciones de exclusión, de unos modelos de masculinidad en los que la agresividad y la violencia cobran especial relevancia, así como la existencia de diferentes circunstancias que fuerzan a las mujeres a iniciar una relación y, de esta manera, las sitúan en un posición de 
espacial vulnerabilidad en ella. Entre los segundos cabe mencionar la ausencia de apoyos familiares y sociales, que incrementa la vulnerabilidad de las mujeres; los efectos de la acumulación de dificultades en distintas esferas; el abuso de drogas por parte del varón; y la presencia de un entorno que, en ocasiones, tiende a no censurar el recurso a la violencia de género. La novedad del análisis aquí realizado reside tanto en el recurso a la noción de exclusión social como en la importancia atribuida a las relaciones de poder de género a la hora de analizar la etiología de la violencia. Es decir, que el papel jugado por los factores antes mencionados se interpreta en todo momento a la luz del trasfondo estructural de relaciones desiguales de género en el que estos operan y en ausencia del cual su efecto sería necesariamente diferente.

\section{PALABRAS CLAVE}

Violencia de género, violencia del compañero íntimo, exclusión social, interseccionalidad, factores de riesgo.

\section{ABSTRACT}

Based on evidence that, in situations of exclusion, the prevalence of genderbased intimate partner violence increases, this paper analyses the dynamics of the link between these circumstances. To this end, 16 in-depth interviews were conducted with women survivors. They revealed two different routes: being in a situation of exclusion could be a triggering factor or a result of such violence. In this paper a detailed analysis of the first route is provided and a series of elements that could be a triggering factor of violence are identified. These are categorised into two groups: on the one hand, factors that condition the process of formation of couples; on the other hand, factors that facilitate the emergence of gender-based intimate partner violence in an already established intimate relationship. Among the first should be noted: the presence, in certain situations of exclusion, of masculinity role models in which aggressiveness and violence gain special relevance; and the existence of diverse circumstances that force women to start a relationship and, in this way, put them in a position of increased vulnerability in it. Among the second should be noted: the lack of family and social support, that increases women's powerlessness; the effects of the accumulation of difficulties in diverse spheres; the male abuse of drugs; and the presence of an environment which, sometimes, does not disapprove the use of gender-based intimate partner violence. The novelty of the analysis performed herein resides in its reference to the notion of social exclusion and in the importance attributed to gender power relations when analysing the aetiology of violence. In other words, at all times the role of the aforementioned factors is interpreted in the light of the structural background of unequal gender relations in which it operates and without which their effect would necessarily differ. 


\section{KEY WORDS}

Gender-based violence, intimate partner violence, social exclusion, intersectionality, risk factors.

\section{INTRODUCCIÓN}

Existe evidencia de que la violencia de género en la pareja, aun siendo un fenómeno que afecta a mujeres de toda clase y condición social, no las afecta a todas por igual (Sokoloff y Dupont 2005), sino que, en determinados colectivos -entre ellos, mujeres en situación de exclusión social-, su prevalencia se incrementa (Damonti 2019).

Partiendo de esta evidencia, hemos analizado las dinámicas de la relación entre la violencia de género en la pareja y la exclusión social. Para ello, nuestra hipótesis de partida ha sido que la mayor prevalencia de la violencia detectada en tales situaciones podía ser el resultado combinado de dos procesos: por un lado, una situación de exclusión social preexistente podría haber contribuido a desencadenar violencia de género; por otro, el hecho de enfrentar una situación de violencia en el marco de la pareja podría ser lo que habría derivado en rutas descendentes hacia la exclusión.

En este artículo analizamos en detalle el primer recorrido. Más en concreto, nuestro objetivo es identificar, mediante entrevistas en profundidad a mujeres supervivientes, qué elementos, vinculados con una situación de exclusión social, pueden favorecer la aparición de violencia y cómo operan. En relación con esto, partimos de la hipótesis de que existen, en tales contextos, elementos de tipo tanto material como simbólico y de nivel tanto individual como colectivo que sitúan a las mujeres en una situación de riesgo intensificado.

Este tema de investigación, si no se trata de forma adecuada, puede llegar a fomentar explicaciones individualistas de la violencia de género o derivar en criminalización de la población excluida. Para evitar que esto suceda, es imprescindible mantener una mirada estructural. Esto significa, por un lado, recordar que la precondición necesaria de la violencia de género es un trasfondo estructural de relaciones desiguales de género; y, por otro, que el origen de los procesos de exclusión no se encuentra en características individuales, sino en desigualdades estructurales inherentes al sistema capitalista (Laparra et al. 2007). El hecho de que una situación de exclusión preexistente pueda facilitar la aparición de violencia de género, por lo tanto, es algo que deberá reconducirse necesariamente al nivel de la estructura: estructura de género, por cómo esta moldea hombres y mujeres determinados y pauta las relaciones; y estructura de clase, por la explotación, las privaciones y la precariedad que esta genera en grupos que ocupan posiciones bajas.

Las reflexiones más interesantes a este respecto se encuentran en los estudios que se ocupan de interseccionalidad (Barrett et al. 2012; Friedemann-Sánchez y Lovatón 2012; Kiss et al. 2012; Stöckl et al. 2011; Peralta, Tuttle y Steele 2010; 
Damant et al. 2008; Sokoloff y Dupont 2005; Sokoloff 2004; Hampton et al. 2003; Jewkes 2002; Collins 2000). Estos, de hecho, parten precisamente de la constatación de que los diferentes sistemas de opresión están interconectados (Collins 2000) y señalan que la posición de los individuos sólo puede comprenderse a partir de su posicionamiento en diferentes estructuras de opresión que se interseccionan.

Tales investigaciones, no obstante, tienen carácter eminentemente teórico (Damant et al. 2008); y, además, se han ocupado de forma casi exclusiva de la intersección entre género y raza/etnia (Choo y Ferree 2010; Sokoloff y Dupont 2005). Resulta, por lo tanto, particularmente relevante tanto efectuar estudios empíricos que se inserten bajo este paradigma como investigar otras intersecciones. A esto se dedica la presente investigación.

\section{UNA REVISIÓN DE LA LITERATURA}

El referente empírico más cercano a nuestro objeto de estudio son las investigaciones que examinan factores de riesgo de violencia de género. Estas han alcanzado un desarrollo considerable, sobre todo en ámbito anglosajón, pero ninguna se ocupa específicamente de la situación de exclusión social.

Esta noción ha alcanzado un éxito creciente en los estudios sobre desigualdad social a partir de los años noventa del siglo pasado como sustituta de la idea de pobreza, objeto de críticas generalizadas por su orientación exclusivamente economicista (Allman 2013; Laparra et al. 2007; Saraví y Makowski 2011; Shabaan 2011; Subirats 2004). De hecho, los cambios sociales que habían empezado a tener lugar -creciente diversificación, complejización y atomización social, por un lado, y progresivo debilitamiento de los factores tradicionales de integración, por otro- empezaron a mostrar con especial fuerza la necesidad de visibilizar otros elementos de desigualdad además del estrictamente económico (Subirats 2004). En consecuencia, visibilizaron también la necesidad de nuevas herramientas de medición y análisis de la desigualdad social. Entre estas se incluye el concepto de exclusión social. Este, dependiendo del contexto, se utiliza con significados profundamente diferentes; existe, sin embargo, cierto acuerdo a la hora de considerarla como un fenómeno de origen estructural (Laparra et al. 2007; Subirats 2005), de naturaleza procesual (Boon y Farnsworth 2011;) y con carácter multidimensional (van Bergen et al. 2014; Boon y Farnsworth 2011; Laparra et al. 2007; Subirats 2005). Más en detalle, aquí nos remitimos a la definición de exclusión que se llegó a consensuar entre los más importantes investigadores de ámbito estatal (Laparra et al., 2007) y que, como puede verse esquematizada en la tabla 1, considera tres ejes fundamentales (económico, político y social-relacional), que a su vez se descomponen en ocho dimensiones (exclusión del empleo, del consumo, política, de la vivienda, de la educación y de la salud, aislamiento social y conflicto social). 
Tabla 1. Las dimensiones de la exclusión social

\begin{tabular}{|c|c|c|}
\hline Ejes & Dimensiones & Aspectos \\
\hline \multirow{3}{*}{ Económico } & $\begin{array}{l}\text { Participación en la produc- } \\
\text { ción }\end{array}$ & Exclusión de la relación salarial normalizada \\
\hline & \multirow{2}{*}{ Participación en el consumo } & Pobreza económica \\
\hline & & Privación \\
\hline \multirow{3}{*}{$\begin{array}{l}\text { Político (ciu- } \\
\text { dadanía) }\end{array}$} & \multirow{2}{*}{ Ciudadanía política } & Exclusión de los derechos políticos \\
\hline & & Abstencionismo y pasividad política \\
\hline & Ciudadanía social & $\begin{array}{l}\text { Acceso limitado a los sistemas de protección } \\
\text { social (sanidad, vivienda y educación) }\end{array}$ \\
\hline \multirow{3}{*}{$\begin{array}{l}\text { Social (rela- } \\
\text { cional) }\end{array}$} & Ausencia de lazos sociales & Aislamiento social, falta de apoyos sociales \\
\hline & \multirow{2}{*}{ Conflicto social, anomía } & Conductas asociales (ej. adicciones) \\
\hline & & Conductas delictivas \\
\hline
\end{tabular}

Fuente: Adaptación de Laparra et al. 2007

La citada ausencia de investigaciones sobre factores de riesgo de violencia de género que utilicen la noción de exclusión, por otra parte, es tanto más llamativa en cuanto varias investigadoras ya han subrayado la necesidad de examinar el impacto cumulativo de varios factores (White et al. 2011), algo que remite de forma muy directa a dicha noción de exclusión. Estos estudios, por el contrario, sí examinan el papel de elementos (falta de formación, desempleo, pobreza, adicciones, etc.) que guardan cierta relación con los procesos de exclusión. La información que aportan, por lo tanto, aun siendo incompleta, nos ayuda a efectuar un primer acercamiento al tema objeto de estudio y resulta, por lo tanto, relevante.

Dentro de esas investigaciones, la mayoría se limita a aportar datos cuantitativos, sin indagar en los mecanismos subyacentes a los mismos (Chan 2014; Mavrikiou et al. 2014; Khalifeh et al. 2013; Vives-Cases et al. 2011; White et al. 2011) y no resulta por lo tanto de interés aquí. Una minoría, sin embargo, sí los examina (Khalifeh et al. 2013; Barrett et al. 2012; Friedemann-Sánchez y Lovatón 2012; Kiss et al. 2012; Stöckl et al. 2011; Peralta et al. 2010; Hampton et al. 2003; Jewkes 2002) y se convierte por ello en un referente privilegiado.

En el caso de los varones, estos estudios identifican dos grandes bloques: el primero defiende que situaciones como pobreza o desempleo pueden desencadenar violencia de género por el estrés y ansiedad que originan (Jewkes 2002; Stöckl et al. 2011). El segundo evidencia que tales situaciones pueden precipitar violencia como respuesta a una sensación de pérdida de control (Peralta et al. 2010; Jewkes 2002), emasculación (Fodor 2006) y crisis de identidad (Peralta et al. 2010; Jewkes 2002). En lo que respecta a las mujeres, estos estudios eviden- 
cian que elementos como un nivel formativo bajo o pobreza suelen acompañarse de menor empoderamiento social, menor integración en redes sociales, menor seguridad en una misma, menor habilidad para utilizar los recursos disponibles en la sociedad e incluso bienestar, todos factores que vulnerabilizan a las mujeres frente a la emergencia de violencia de género (Stöckl et al. 2011; Lanier y Maume 2009; Jewkes 2002). Otras investigaciones, finalmente, evidencian que, en ciertas situaciones (como la falta de formación), podría haber menores capacidades para un manejo no violento de los conflictos (Friedemann-Sánchez y Lovatón 2012), así como una mayor adhesión, en varones y en mujeres, a valores patriarcales y creencias más tradicionales acerca de los roles de género (Flood y Please 2009; Hunnicutt 2009; Ahmad et al. 2004).

Un último elemento a resaltar es que la gran mayoría de estos estudios carecen de perspectiva de género (Chan 2014; Mavrikiou et al. 2014; GonzalezGuarda 2013; Renner y Whitney 2012; van Vijk y de Bruijn 2012; White et al. 2011; Cunradi et al. 2011; Panchanadeswaran et al. 2010; Raj y Silverman 2002); y que incluso las investigaciones que sí consideran el papel del género en la etiología de la violencia reservan un papel muy limitado al análisis del mismo (Franklin y Menaker 2014; Strier et al. 2014; Nóblega-Mayorga 2012; Peralta et al. 2010; Jewkes 2002; Yick 2001). En la presente investigación, por el contrario, el papel de la situación de exclusión como factor precipitante de la violencia se comprende siempre en relación con la desigualdad y las relaciones de poder de género subyacentes. Únicamente así, de hecho, puede entenderse por qué los elementos de riesgo, aun afectando tanto a hombres como a mujeres, desencadenan violencia masculina contra la pareja femenina pero no lo contrario.

\section{METODOLOGÍA}

Como ya se ha apuntado, partiendo de la evidencia de que, en situaciones de exclusión social, la prevalencia de la violencia de género en la pareja se incrementa (Damonti 2019), hemos querido identificar qué elementos, vinculados con tales situaciones, pueden favorecer la aparición de esta violencia y cómo operan.

Para ello, partimos de la hipótesis de que existen factores, de tipo tanto material como simbólico y que actúan tanto a nivel individual como relacional y colectivo, que sitúan a las mujeres en situación de exclusión en un contexto de riesgo intensificado. También avanzamos la hipótesis de que estos elementos operan en dos momentos clave: (1) en el proceso de formación de pareja, favoreciendo que las mujeres inicien unas relaciones en las se hallan en peligro desde el primer momento; y (2) a lo largo de la relación, facilitando que esta violencia vaya apareciendo con el paso del tiempo.

En relación con estas hipótesis, hemos analizado en profundidad dos elementos: (1) el proceso de formación de pareja (ahondando en cuestiones como las circunstancias en que la mujer y su pareja se conocieron, cómo era la vida de la mujer en ese momento, qué significó para ella conocer a esa persona, qué la llevó a iniciar una relación con él, si considera que en otras circunstancias 
habría tomado otras decisiones, etc.); y (2) la historia de la violencia (examinando cuestiones como el contexto en el que esta empezó a manifestarse, si había habido algún cambio con respecto a la situación anterior, si había alguna circunstancia en la que la violencia se volvía más intensa, cuál era la reacción de la mujer a la misma, si considera que, en otras circunstancias, esta podría haber sido diferente, etc.).

Para abordar específicamente el análisis de estos elementos, hemos recurrido a una metodología cualitativa, caracterizada por permitir un acceso profundo a la experiencia de los sujetos (Flick 2015). Más concretamente, hemos realizado entrevistas en profundidad a mujeres supervivientes, una técnica de análisis que permite recoger una información subjetiva y rica (Kvale 2011) y que, en este caso, favorece la interpretación, la clarificación y la elaboración de sentido para la relación entre exclusión y violencia. Más en detalle, escogimos entrevistar tanto a mujeres que viven o han vivido procesos de exclusión de diferente tipo e intensidad como otras en situación de integración (con fines de control).

Para establecer con más precisión el perfil de las mujeres a entrevistar, hemos utilizado dos criterios diferentes. En una primera fase, realizada en 2013, la necesidad de garantizar la máxima variabilidad de la muestra nos llevó a considerar una serie de variables de tipo sociodemográfico. El primer análisis así realizado mostró un efecto de saturación en relación con algunos aspectos examinados, pero no en otros (entre ellos, el que aquí nos ocupa). Consecuentemente, en 2015, se realizó una segunda fase de recogida de información, dirigida específicamente a buscar perfiles que permitiesen dilucidar aquellos aspectos en relación a los cuales todavía faltaba información. Esta decisión es coherente con el método de muestreo gradual de Glaser y Strauss, donde las decisiones sobre la selección y reunión del material empírico se toman durante el proceso de recogida y análisis de los datos (Flick 2015).

El contacto con las mujeres se ha realizado a través de recursos tanto públicos como del Tercer Sector y tanto de ámbito urbano como rural de Navarra. Más concretamente, las supervivientes entrevistadas han sido derivadas: del Servicio Municipal de Atención a la Mujer del Ayuntamiento de Pamplona; de Cruz Roja Navarra; del Área de Mujer e Igualdad del Ayuntamiento de Estella; del Servicio Social de esta misma ciudad; de la Asociación pro derechos de la mujer maltratada; de la Confederación Española de Personas con Discapacidad Física y Orgánica; y de una comunidad terapéutica que no nombramos para evitar la posible identificación de la mujer entrevistada. A través de la mayor variedad de los canales de contacto, hemos pretendido reducir el sesgo de selección.

Una vez establecidos los contactos, hemos procedido a la realización de las entrevistas, 11 en una primera fase y 5 en la segunda.

En lo que respecta a cuestiones éticas, cabe señalar que, en la realización de las entrevistas, el bienestar de las informantes ha sido en todo momento prioritario frente al objetivo de la recogida de información; que, en todos los casos, las supervivientes accedieron expresamente a participar en la investigación y a que la entrevista fuese grabada para facilitar el análisis, firmando para ello un documento de consentimiento informado; y que, a la hora de utilizar la información, 
ha sido prioritario garantizar en todo momento el anonimato de las informantes, razón por la cual todos los nombres propios que aparecen en el texto han sido sustituidos por pseudónimos y se ha modificado asimismo cualquier otro dato que pudiese conducir a su identificación.

Se presenta ahora una tabla de resumen en la que se explicitan las características sociodemográficas básicas de las mujeres entrevistadas y se efectúa asimismo una aproximación a su situación social, señalando si, al principio de la relación y a lo largo de la misma, estas se hallaban en situación de integración, precariedad o exclusión social y, para las que se hallaban en situación de precariedad o exclusión, señalando también los principales elementos de vulnerabilidad social identificados. En lo que a esto respecta, la referencia es la noción de exclusión detallada en la tabla 1, adaptada a las necesidades de la presente investigación y de un análisis de tipo cualitativo. En relación con ello, los principales elementos de vulnerabilidad considerados son un bajo capital cultural, un empleo precario o inexistente, pobreza económica y privaciones, precariedad en el ámbito de la vivienda, falta de redes familiares y sociales, mala salud y situaciones de drogodependencia. En lo que respecta a la historia de vida, además, elementos clave son una experiencia de violencia de género en la familia de origen y embarazos adolescentes. A partir de aquí, la intensidad con la que se presenta cada factor de vulnerabilidad, el mayor o menor número de factores detectados y las interrelaciones entre ellos son las variables clave para situar a una mujer en el espacio de la integración, precariedad o exclusión. No se trata, en cualquier caso, de una fórmula matemática, sino de una directriz general que deberá ser interpretada caso por caso a partir de los relatos de las supervivientes y de la sensibilidad de las investigadoras. 


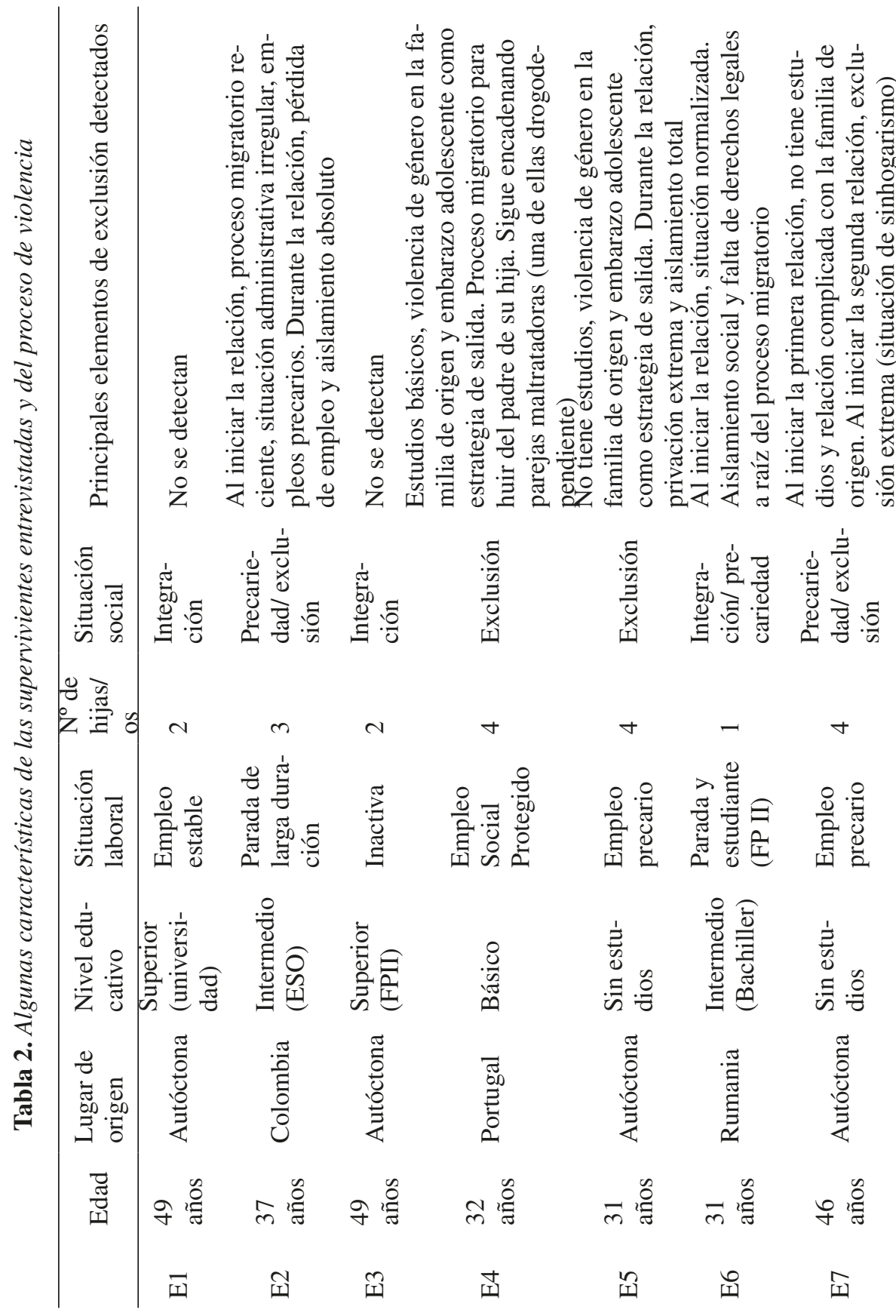



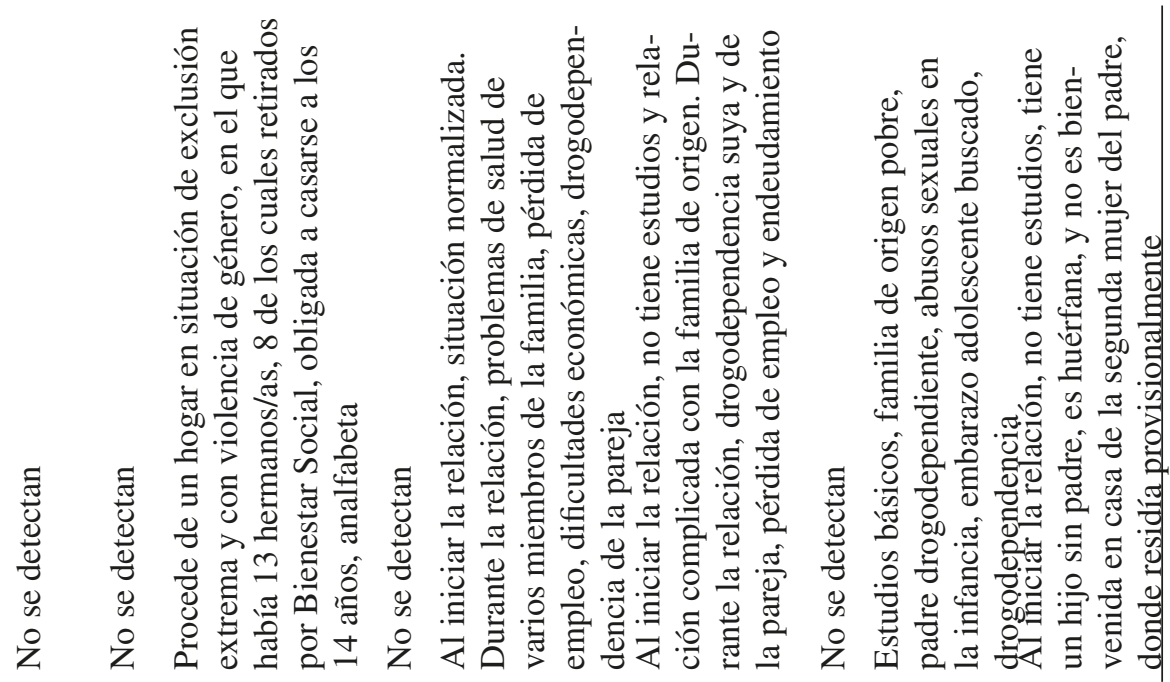

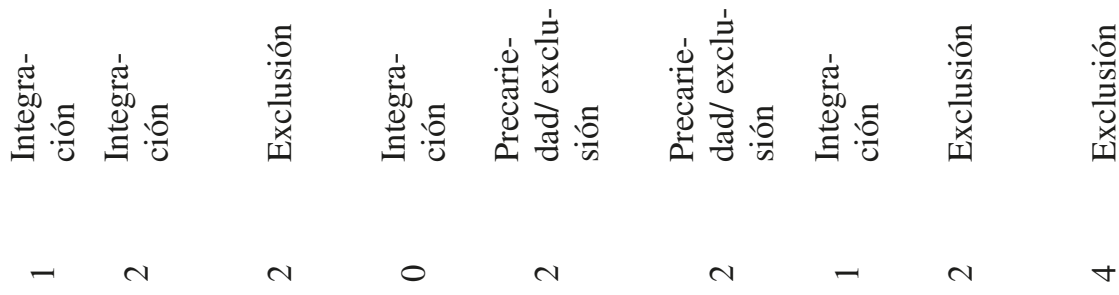

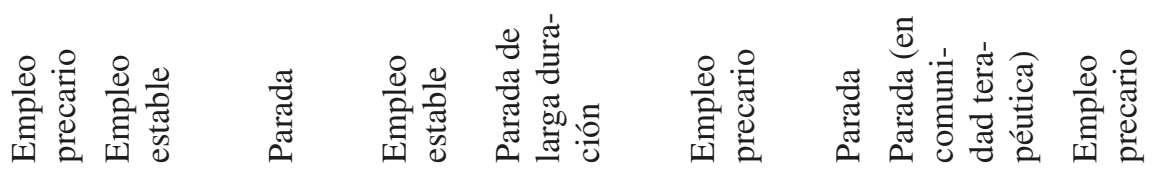

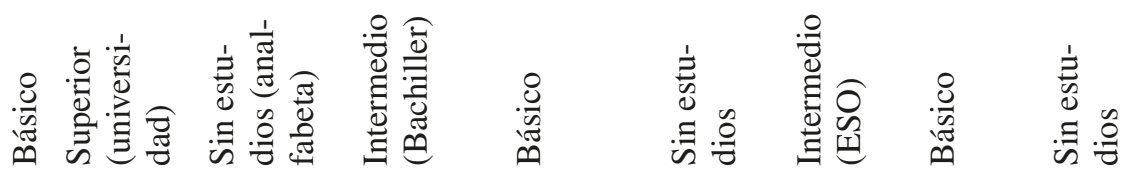

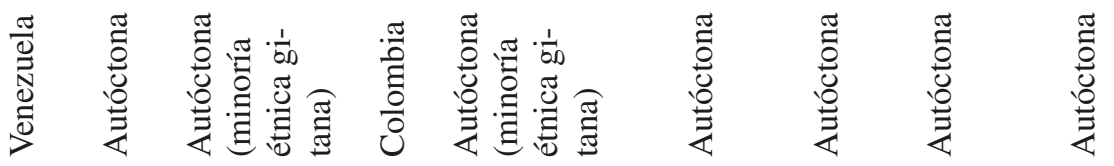

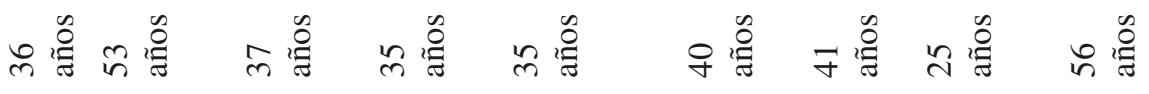

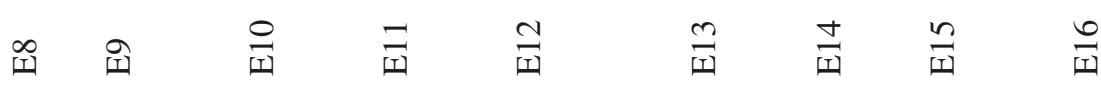


Todas las entrevistas han sido grabadas y trascritas de manera literal. Al finalizar cada una hemos realizado, además, un registro escrito, anotando las primeras impresiones recabadas así como los elementos más significativos en relación con el objeto de estudio.

El análisis del corpus textual se ha realizado con el auxilio del programa informático Atlas.ti.6.1.1, herramienta mediante la que se procedió a la segmentación y codificación del texto. Los códigos utilizados, en algunos casos, procedían de la identificación de elementos claves de la revisión bibliográfica y, en otros, fueron establecidos de manera emergente a lo largo del análisis. En un segundo momento, los códigos así identificados se revisaron, jerarquizándolos, depurándolos y ajustando la codificación previa. En tercer lugar, a partir de la agrupación de códigos se estableció el sistema categorial de análisis definitivo, el que aquí se presenta, en función de su solidez conceptual y con un mayor nivel de abstracción (Kuckartz 2014). Este procedimiento permite sistematizar el contenido del corpus textual para la detección de reiteraciones que saturan los campos de significado y asegura un mayor rigor en el análisis.

En este sentido, el proceso de análisis se articuló en torno a dos grandes ejes que incluían, en primer lugar, categorías referidas a la formación de pareja: como el emparejamiento temprano, forzado por condiciones materiales de vida, o la intensificación y refuerzo contextual de los rasgos de agresividad en los modelos de masculinidad. En segundo lugar, se han identificado categorías referidas a factores que facilitan la aparición y la intensificación de la violencia: como la ausencia de apoyos familiares y sociales de las mujeres, la acumulación de dificultades en diferentes esferas, los consumos y adicciones del varón y la normalización de grados intensos de violencia por parte del contexto.

\section{RESULTADOS Y DISCUSIÓN}

Como ya se ha apuntado, a partir del análisis así realizado, e inscrita en el marco estructural de relaciones de género, la primera distinción se establece entre factores que condicionan el proceso de formación de pareja y otros que inciden en las dinámicas de la interrelación entre los dos miembros de la misma, aumentando la probabilidad del recurso a la violencia por parte del agresor. Evidentemente, una diferenciación tan clara entre estos dos grupos responde a necesidades analíticas -nos ayuda a aislar elementos y crear un esquema explicativo- pero la realidad es infinitamente más compleja y las interrelaciones entre los dos grupos de factores estrechas y frecuentes.

\subsection{Factores que condicionan el proceso de formación de pareja}

Para empezar, centramos la mirada en los factores que condicionan el proceso de formación de pareja, favoreciendo que las mujeres, empujadas por las 
situaciones que viven, comiencen unas relaciones en las que se hallan en peligro desde el primer momento.

\subsubsection{Unas condiciones materiales de vida que "fuerzan" a las mujeres a iniciar una relación}

En primer lugar, para comprender qué especificidades tiende a adquirir el proceso de formación de pareja en situaciones de exclusión y cómo esto puede situar a las mujeres en una situación de riesgo intensificado, hay que considerar las condiciones materiales de vida en las que se encuentran estas mujeres (y el impacto que esto tiene también en la esfera emocional). En este sentido, un elemento clave que aparece en el relato de varias de las supervivientes en situación de exclusión entrevistadas es la inseguridad residencial. Con ello, se hace referencia a la realidad de mujeres que no tenían una vivienda propia ni se sentían bienvenidas en aquella en la que provisionalmente residían y que vieron en el hecho de formar una pareja la única estrategia de salida a su alcance. Se trata de un elemento de gran importancia y que aparece con gran frecuencia en las entrevistas y que, sin embargo, no es señalado por la literatura.

Es este, por ejemplo, el caso de Concepción, cuya madre murió siendo ella una niña y que nunca llegó a establecer una buena relación con la nueva mujer del padre. Siendo ella adolescente, además, este también murió y, al quedarse ella embarazada, la madrastra la envió a un instituto religioso para madres solteras. Un tiempo después, incapaz de seguir soportando la realidad de aquel centro, volvió a la casa paterna, pero no era bienvenida allí y era consciente de ello. En ese contexto, conoció a un hombre (que la maltrataría desde el primer momento y durante décadas) y enseguida se fue a vivir con él, no porque mediase un fuerte enamoramiento de por medio, sino porque, como ella misma relata, no tenía otro lugar en el estar:

Empecé a salir con él, pues por eso, por... porque yo no tenía sitio (...) pero... vamos que yo no... yo no quería eso (E16)

Para comprender plenamente el impacto de las condiciones materiales de vida, además, es importante recordar que la precariedad de las mismas (y, más concretamente, el hecho de no poder contar con una vivienda propia, viéndose obligadas a buscar cobijo en lugares donde no se es bienvenidas) no tiene un efecto puramente práctico, sino que incide también en la esfera emocional. Esto se puede apreciar en el caso de Sheila que, en su relato, vincula claramente el malestar emocional, resultante del hecho de no disponer de una vivienda propia y del rechazo manifestado por sus padres, con su decisión de iniciar una relación (y muy rápidamente una convivencia) con un hombre que desde el primer momento ejerció violencia contra ella:

Acababa de volver de un centro (...) mi madre no quería saber nada de mí... (...) mi padre me dejó quedarme en su casa, pero como... "sí te dejo porque eres mi hija y..." ¿sabes?, "no me queda otra...". (...) estaba baja... 
necesitaba cariño como sea. Como sea. Y... y lo fui a buscar en lo menos indicado (E15)

Análogamente, también cabe señalar varios casos de mujeres que se vieron empujadas a formar una pareja como forma de huir de un hogar inseguro, en el que había violencia de género. Nuevamente, se trata de un elemento de importancia considerable y que, sin embargo, los estudios sobre factores de riesgo consultados no contemplan. Es este, por ejemplo, el caso de una de las mujeres entrevistadas, que, siendo adolescente, vio en el embarazo la única estrategia a su alcance para lograr salir de la situación en la que se encontraba:

Mira, yo me quedé embarazada, por... salir de mi casa, la verdad es que me quedé embarazada por salir de mi casa, porque... también m... mi madre, ha sido una mujer maltratada (...) la única solución, que yo en esos tiempos [con 16 años] veía, me quedé embarazada (E4)

El fragmento presentado también refleja otra cuestión de gran importancia: el hecho de que, con gran frecuencia, para las mujeres en situación de exclusión social entrevistadas formar pareja y quedarse embarazadas, a menudo siendo todavía adolescentes o, en cualquier caso, muy jóvenes, se viera como una estrategia válida y eficaz para salir de la situación en la que se encontraban. El hecho de que el embarazo se percibiese como una opción apetecible en edades tan tempranas es algo que, como señala la literatura (Elley 2011), está claramente marcado por la posición de clase. En contextos de clase baja (y aún más en situaciones de exclusión social), de hecho, el horizonte vital de las chicas jóvenes se encuentra fuertemente limitado de antemano y esto favorece que, en tales contextos, la decisión de quedarse embarazada, lejos de percibirse como una gran renuncia a todo lo que la vida podría ofrecer y una limitación de las aspiraciones vitales, tienda a interpretarse más bien como una herramienta que puede otorgar una nueva identidad y respetabilidad, así como un lugar social propio.

\subsubsection{Unos modelos de masculinidad en los que la agresividad y la violencia cobran especial relevancia}

Un segundo elemento que aparece en el relato de varias de las mujeres en situación de exclusión entrevistadas -y que condiciona con quién se establece el vínculo- guarda relación con algunas características específicas que el modelo hegemónico de masculinidad tiende a adquirir en tales contextos. En lo que a esto respecta, hay que considerar que los elementos clave de la masculinidad hegemónica - la fuerza, la valentía, la competitividad, la agresividad, la seguridad en sí mismo, etc. (Lagarde 2008) - constituyen, como señala precisamente la investigación con perspectiva de género, un germen fundamental del ejercicio de violencia contra las mujeres. Este modelo está presente en todas las clases y condiciones sociales, pero -y esto es lo que aquí nos interesa reseñar- no se manifiesta de forma idéntica en todas las circunstancias, sino que puede asumir características diferentes dependiendo del contexto social y las oportunidades, 
tanto materiales como simbólicas, que en él se encuentren. Se trata de algo que la literatura, principalmente anglosajona, ha investigado en detalle, descubriendo que, en contextos de exclusión social, la imposibilidad de acceder a los recursos clave para alcanzar una masculinidad dominante en términos clásicos ha influido en una redefinición de la misma, donde el recurso a la violencia, en general, adquiere una importancia elevada (Strier et al. 2014; Friedemann-Sánchez y Lovatón 2012; Peralta et al. 2010; De Kaseredy y Schwartz 2005; Hampton et al. 2003; Jewkes 2002). Es fácil comprender cómo esto, al interseccionarse con una estructura social patriarcal, puede facilitar también procesos de violencia de género.

Lo que la literatura apenas ha señalado, sin embargo, es que esta eventual caracterización diferente de la masculinidad en función del contexto social no impacta únicamente en la configuración subjetiva de los hombres, sino que incide también en la de las mujeres, modulando las cualidades que estas tienden a valorar en un posible compañero. Se trata de algo fácilmente comprensible: por un lado, de hecho, tanto las unas como los otros se han socializado en el mismo mundo y esto incrementa las posibilidades de encuentro. Por otro lado, además, hay que considerar también que, en un contexto en el que las condiciones materiales de vida son muy duras, la idealización de una figura masculina protectora -presente, por otra parte, en el imaginario amoroso que impregna las relaciones desiguales de género (Illouz 2012)-, que presente específicamente rasgos de fuerza y agresividad, es comprensible, aunque pueda suponer un elemento de vulnerabilización relacional. Todo esto se aprecia claramente en el relato de una de las mujeres entrevistadas, que señala que, en un primer momento, lo que más le atrajo de su pareja fue justamente su agresividad, que ella relacionaba con fuerza y capacidad de protegerla (algo que, a posteriori, como ella misma reconoce, se convierte en un factor de riesgo muy claro):

¿Al principio, qué me gustaba de él? (...) era muy chulito, muy "aquí estoy yo", ¿sabes? Y eso, a mí siempre me... me ha tirado un tipo duro, ¿no? (...) me sentía protegida, me sentía que ya nadie me iba...ya nadie se iba a meter conmigo, más que si llevaría un pitbull al lado. Porque iba el tiarrón este y... y mira, total que... [por el tono, se entiende que está haciendo referencia al hecho de que realmente era de él de quién habría necesitado protección, aunque entonces no lo sabía]... (E15)

Eso lleva en ocasiones a las mujeres a iniciar relaciones con hombres particularmente agresivos y violentos, en general y también con ellas. De hecho, en los procesos de violencia en contextos de exclusión es más frecuente que los agresores ejerzan esta violencia tanto dentro de casa como fuera, tanto contra la pareja femenina como contra otras personas. En contextos de integración esto es menos frecuente, dado que los agresores tienden a serlo solo contra la pareja y las y los menores, manteniendo, sin embargo, un comportamiento adecuado fuera de ese marco. El hecho de que, en contextos de exclusión, la violencia y la agresividad tiendan a trascender las fronteras de la pareja y del hogar es algo que 
aparece, por ejemplo, en el relato de Elena que, en relación a su ex marido y la familia de él, señala que:

Nadie se atreve a meterse con esta gente, para nada, porque saben, lo agresivos que son. Y que, si te pones con ellos, pues te pegan (...) tanto él como los hermanos (...) son personas conflictivas con el resto de la gente (E5)

\subsection{Factores que facilitan la aparición de violencia de género en una pareja ya constituida}

Como se ha señalado más arriba, para comprender la mayor vulnerabilidad a la violencia de género de las mujeres en situación de exclusión social, analizar el proceso de formación de pareja no es suficiente, sino que hay que examinar asimismo el papel de aquellos elementos que pueden desplegar sus efectos a lo largo de toda la relación, facilitando el desencadenamiento de violencia de género en una pareja ya constituida.

\subsection{1, Ausencia de apoyos familiares y sociales}

Un primer elemento de riesgo identificado es la ausencia de apoyos familiares y sociales. Esta es una de las dimensiones clave de los procesos de exclusión (Laparra et al. 2007).

Lo que aquí interesa resaltar es que esta falta de apoyos es muy relevante para la comprensión del origen y desarrollo de los procesos de violencia, puesto que se trata de algo que puede contribuir a erosionar las barreras a su utilización. Desde una mirada relacional a la violencia, de hecho, se comprende que, en un proceso incipiente de aparición de la misma, la falta de recursos y redes con los que la mujer puede contar juega un papel relevante, no sólo en la posibilidad de alejamiento y respuesta de ella, como veremos, sino también en las dinámicas que este proceso probablemente seguirá. El hecho de saber que la mujer no tiene quien la respalde, que no hay nadie que la pueda apoyar y sostener, ni nadie que pueda acudir en su ayuda, de hecho, es percibido por el agresor, condicionando -de forma consciente o inconsciente- el avance del proceso de violencia y facilitando su paulatina intensificación:

Entonces, él se aprovecha por eso, y se piensa que: «bueno, como su familia también le ha dado de lado, la tienen tiradita, pues aquí aprovecho yo" (...) y ya pues, te la lía (E12)

Paralelamente, esta falta de apoyos familiares y sociales modula también la capacidad de respuesta de las mujeres, limitando sus opciones de respuestas a las primeras manifestaciones de la violencia, algo que retroalimenta el proceso descrito. Esta situación de especial indefensión emerge claramente en el relato de varias de las mujeres supervivientes, entre ellas, por ejemplo, Manuela. Esta 
mujer, de hecho, señala cómo el hecho de no contar con ningún tipo de apoyo -a los 16 años decidió quedarse embarazada precisamente para poder alejarse de su familia de origen y luego se vio abocada a abandonar su país, perdiendo todo contacto, para huir de la violencia que ejercía contra ella el padre de su hija- reducía fuertemente su margen de maniobra en una nueva relación que había iniciado y, en relación con ello, también sus opciones para oponerse a la violencia que vivía en ella:

Yo tenía que hacer lo que él quería, porque yo estaba totalmente isolada, del mundo (E4)

Si en los ejemplos mostrados esta falta de apoyos estuvo presente desde los primeros tiempos de la relación, en otros de los casos analizados el aislamiento social es algo que aparece en un segundo momento, a raíz de un proceso migratorio, y es justamente allí cuando la violencia aparece o se intensifica. En este sentido, es emblemática la historia de vida de Nicoleta, que empieza a experimentar violencia de alta intensidad después de años de relación, cuando llega al Estado español con un permiso de reagrupamiento familiar solicitado por el marido. Al llegar, se encuentra en un país nuevo, en el que no puede contar con ninguna red familiar ni social, cuyo idioma desconoce y en el que no tiene permiso de trabajo (algo que, además de impedirle cualquier tipo de independencia económica, también le imposibilita poder establecer relaciones con compañeras y compañeros de trabajo al margen del marido). En este contexto, la violencia de baja intensidad que el marido ya ejercía contra ella se recrudece fuertemente:

Él ha venido con contrato de trabajo. (...) luego a partir de tres meses, pues él me ha hecho invitación para venir con la hija, y vivir con él la... en la España. Y he venido, y... las cosas se ha empeorado, porque yo... no sabía... hablar, no tenía muchos, conocidos, (...) no tenía permiso de trabajo (...) Ningún tipo de apoyo. Sólo tenía amistades, sus amigos. Y no podía, hablar con ellos (E6)

La vulnerabilidad que una situación de aislamiento social conlleva, por otra parte, es algo que la literatura especializada en violencia de género implícitamente reconoce cuando señala que todos, o casi todos, los agresores, en los primeros estadios del proceso de violencia, intentan justamente separar a las mujeres de sus contactos familiares y sociales y que lo hacen precisamente con el fin, consciente o inconsciente, de aumentar su vulnerabilidad y dependencia de ellos (Jewkes 2002; Ruíz-Jarabo y Blanco 2007).

\subsubsection{Acumulación de dificultades en distintas esferas}

Otro factor que puede facilitar la precipitación de procesos de violencia de género es la presencia de elementos estresores externos. Se trata de un elemento que aparece en las investigaciones consultadas, pero estas tienden a resaltar el papel jugado por dificultades en ámbitos puntuales, como una situación de des- 
empleo (Stöckl, Heise y Watts 2011) o de pobreza económica (Jewkes 2002). En nuestro caso, al contrario, los relatos de las supervivientes entrevistadas muestran un panorama más complejo, en el que lo que actúa como desencadenante no es la aparición de problemas en un único ámbito, sino la acumulación y retroalimentación de dificultades en distintas esferas. Se trata, por otra parte, de una realidad estrechamente vinculada con procesos de exclusión, puesto que estos han sido definidos justamente como la incidencia simultánea, en diferentes ámbitos, de distintos elementos de vulnerabilidad que se suman, se interrelacionan y se retroalimentan (Gabàs I Gasa 2003).

El papel precipitante de esta acumulación de dificultades aparece con claridad en el relato de Carmen que, según ella misma relata, durante los primeros diez años de matrimonio no experimentó violencia a mano de su marido, hasta que la aparición concomitante de graves problemas en ámbitos muy distintos contribuyó a desencadenarla. Según su relato, de hecho, cuando la relación ya había empezado a complicarse con el nacimiento del segundo hijo, la familia tuvo que enfrentar graves dificultades a nivel de salud (se reactivó la enfermedad hereditaria degenerativa que ella padecía, descubrieron que el hijo pequeño también la tenía y, contemporáneamente, el padre y la cuñada del varón enfermaron de cáncer); todo esto tuvo también fuertes repercusiones económicas, dado que ella se vio obligada a dejar el empleo y, como resultado, ya no pudieron mantenerse al día con los gastos de hipoteca; la pareja, mientras tanto, había empezado a consumir cocaína de forma descontrolada. Todo esto precipitó un proceso de violencia de género:

La relación muy bien, los dos trabajando (...) todo muy bien muy bien muy bien. Luego... según pasaban los años (...) tuve al crío pequeño, y a raíz de allí ya (...) la relación se empezó a complicar. Luego, su padre, empezó con cáncer, una cuñada suya también, pa' colmo mi enfermedad (...) y ya, para... la gota que colmó el vaso fue el crío, el crío también la misma enfermedad. Luego, pues, yo dejé de trabajar, se nos acumularon los gastos de la hipoteca, bueno... todo eso... Pues él, nunca le faltaba la cocaína en el bolsillo, para el trabajo y para todo y... y eso fue ya... (...) se nos vino todo encima, y todo tan seguido... (...) y ya no se pudo aguantar más (E12)

Es importante señalar, sin embargo, que si la acumulación de dificultades en muy distintos ámbitos ciertamente tuvo un peso muy significativo a la hora de desencadenar la violencia, no podemos, sin embargo, olvidar que el hecho de que tales dificultades hayan derivado en un proceso de violencia masculina contra la mujer y no lo contrario sólo puede entenderse si se comprende dicha violencia en relación a la desigualdad y las relaciones de poder de género. Se trata de un matiz clave que los estudios consultados, sin embargo, tienden a ignorar. 


\subsubsection{Abuso de alcohol u otras drogas por parte del varón}

El hecho de que alguien en el hogar tenga alguna drogodependencia es otro factor de exclusión (Laparra et al. 2007) y, a la vez, algo que, aun sin ser causa de violencia de género, puede actuar como desencadenante puntual. Que el consumo de alcohol y otras drogas sea un factor de riesgo es algo ampliamente reconocido por la literatura (ej. Barrett, Habibov y Chernyak 2012; Grose y Grabe 2014; Kiss et al. 2012; Lanier y Maume 2009; Stöckl, Heise y Watts 2011) y que se vincula con varios elementos, como: los conflictos que de él se puedan derivar (ej. Barrett, Habibov y Chernyak 2012; Jewkes, Levin y Penn-Kekana 2002; Stöckl, Heise y Watts 2011); la reducción de las inhibiciones y de la capacidad de controlar de los impulsos (Barrett et al. 2012; Jewkes 2002); el hecho de que, socialmente, estar bajo el efecto de sustancias estupefacientes se ha considerado una "excusa" aceptable para el comportamiento violento (Jewkes 2002); o el hecho de que, en determinadas circunstancias en las que la masculinidad de percibe amenazada (ej. desempleo), el consumo de drogas y las conductas violentas podrían ir unidos porque responden ambos al objetivo de restablecer esa masculinidad dominante que peligra (Peralta et al. 2010). Se trata, en cualquier caso, de algo que aparece con gran frecuencia en el relato de las mujeres en situación de exclusión entrevistadas, entre ellas, por ejemplo, Cristina, que refiere cómo la primera agresión física se dio justamente en un momento en el que el varón estaba bajo el efecto de sustancias estupefacientes:

La primera vez que me tocó, fue la... la del coche (...) él iba hasta el culo, el tío. Iba, puesto totalmente (E13)

En otros casos, lo que incrementa el riesgo de agresión no es el consumo de drogas en sí mismos, sino el hecho de no tener acceso a ellas cuando se tiene deseo (o necesidad) de hacerlo. Se trata de un elemento de gran relevancia y estrechamente vinculado con situaciones de exclusión y del que no se encuentran referencias en la literatura especializada:

Una por una, que él... que a él no le faltara pa' consumir (...) y mismo yo no me permitía que a él le faltara a él pa' consumir, porque sabía yo lo que iba a pasar. Es que, día que no consumía, palizón que llevabas (E4)

En cualquier caso, es importante resaltar que el consumo de alcohol u otras drogas no es una causa directa de la violencia, sino un simple elemento desencadenante que, si actúa como lo hace, es porque se inserta en la lógica relacional del sistema de sexo/género. Se trata de una apreciación clave -solamente así, de hecho, se puede comprender por qué el consumo de drogas deriva en violencia masculina contra la pareja femenina y no lo contrario- que, sin embargo, los estudios sobre factores de riesgo consultados tienden a no considerar.

En relación con esto, también cabe destacar que, habitualmente, el hecho de que la pareja haya o no consumido drogas no guarda relación con la presencia o ausencia de violencia, sino con su intensidad. Es decir, que el hecho de en- 
contrarse bajo los efectos de sustancias estupefacientes, más que desencadenar violencia, la intensifica:

Él, no le hacía falta estar borracho para decir que «puta». ¿No? O para decir que «esto no sabes hacerlo así», o para decirte, yo que sé... para eso no le hacía falta estar borracho, ¿vale? Pero si es cierto que cuando venía borracho era peor (E5)

Se trata de un elemento de gran importancia y que, sin embargo, los estudios consultados no remarcan, muy probablemente porque se trata de investigaciones de tipo cuantitativo y que, además, en muchos casos (ej. Kiss et al. 2012; Lanier y Maume 2009; Stöckl, Heise y Watts 2011) no contemplan las manifestaciones psicológicas de la violencia.

\subsubsection{Un entorno que no censura el recurso a la violencia de género}

Finalmente, un último elemento que aparece en el relato de varias de las mujeres en situación de exclusión social entrevistadas es el hecho de que ni el entorno social de sus parejas ni el suyo propio han llegado jamás a reprobar la violencia que sus parejas ejercían contra ellas, algo que guarda probablemente relación con algunas cuestiones señaladas por la literatura, como la presencia, en contextos de exclusión, de valores más fuertemente patriarcales y creencias más tradicionales acerca de los roles de género (Flood y Please 2009; Hunnicutt 2009; Ahmad et al. 2004) y una mayor aceptación de la violencia como elemento fundante de la masculinidad (Strier et al. 2014; Friedemann-Sánchez y Lovatón 2012; Peralta et al. 2010; De Kaseredy y Schwartz 2005; Hampton et al. 2003; Jewkes 2002).

Esta falta de reprobación del entorno puede apreciarse a partir de varios elementos, como el hecho de que esta violencia no se ejerciera únicamente en ámbitos privados, sino también delante de otras personas (incluso en sus formas brutales), en una clara demostración de que ocultarla simplemente no era necesario:

Paliza y... y otra, y otra, y otra, vivía su sobrino enfrente, le daba igual, delante de su sobrino, de su novia, de... es que le daba igual, hasta delante de sus hermanas, delante de... que le daba igual (E15)

Otro elemento que refleja esta falta de reprobación del entorno es que, a menudo, cuando las mujeres llegan finalmente a poner nombre a lo que vivieron y deciden separarse y poner denuncia, sus propias familias se ponen del lado del agresor (y esto pese al hecho de que se tratara de procesos de violencia de altísima intensidad):

Mi hermano (...) cuando me separé de él, me dijo que... que yo tenía la culpa (...) de hecho, tiene trato con él todavía (...) y la casa de mi hermano está abierta para él (...) Ponerle la denuncia, y divorciarme, y ya el otro llamándome, que cómo le he podido hacer eso... que le he tirado la vida abajo... 
que le he amargado la vida... (E13)

Entre las mujeres en situación de integración entrevistadas, por el contrario, en ningún caso hay evidencia de que la violencia se ejerciera delante de otros, ni de que las familias tuvieran conocimiento de la misma o la respaldasen.

Se trata de un elemento que la literatura no señala y que, sin embargo, parece tener una importancia clave en la comprensión del origen y desarrollo de los procesos de violencia de género. El hecho de que el entorno sea más comprensivo con el recurso a la violencia, de hecho, da lugar a una situación que hemos definido de impunidad aprendida -por paralelismo con la noción de indefensión aprendida de Seligman (1975), retomada por Walker en su análisis del ciclo de la violencia (1984) - que favorece tanto el recurso a la violencia como su progresiva intensificación. Más en detalle, si la conocida noción de indefensión aprendida es utilizada para indagar y comprender la permanencia en relaciones violentas de las mujeres que, en un proceso complejo de interacción con el agresor, van intensificando su experiencia de indefensión y debilitando sus defensas psicológicas, producto de la violencia y de sus fallidos intentos por controlarla, con la noción de impunidad aprendida pretendemos ampliar el foco y desplazar la mirada hacia los hombres que ejercen violencia. Se trata de un desplazamiento necesario, puesto que, como señala Bonino (2007: 199), la violencia de género, aun constituyendo un problema "para" las mujeres, no es, no obstante, un problema "de" ellas, sino " $d e$ " la cultura masculina/patriarcal y "de" los hombres. En este sentido, y derivado del análisis de los testimonios, se puede apreciar que los varones agresores experimentan un proceso análogo al que Walker (1984) describía en referencia a las mujeres, con la diferencia de que lo que aprenden no es la indefensión sino la impunidad. Con cada acto violento (desde los más pequeños y sutiles hasta los más intensos) que no es sancionado, con cada agresión (de la intensidad que sea) que no es censurada, de hecho, experimentan un aprendizaje de impunidad, que no solamente favorece la perpetuación del maltrato, sino también su progresiva y paulatina intensificación.

\section{CONCLUSIONES}

En esta investigación hemos tratado de explorar los procesos de violencia de género vividos por mujeres en situación de exclusión. En relación con ello, nuestra hipótesis de partida era que, aunque la violencia de género es un fenómeno de origen estructural que, como tal, puede afectar a mujeres de cualquier clase y condición social, las situaciones de exclusión se asocian a una serie de elementos que pueden facilitar su aparición e intensificación (Damonti 2017), por lo menos en sus formas más evidentes.

A partir del análisis efectuado, efectivamente, hemos podido identificar una serie de factores de naturaleza diversa que actúan en ese sentido; por un lado, condicionando el proceso de formación de pareja y, por otro, incidiendo en las dinámicas de la interrelación entre los dos miembros de la misma. Entre los 
primeros cabe señalar: la existencia de diferentes circunstancias vinculadas a la situación de exclusión que fuerzan a las mujeres a iniciar una relación y, de esta manera, las sitúan en una posición de especial vulnerabilidad en ella; así como la existencia, en determinadas situaciones de exclusión, de unos modelos de masculinidad en los que la agresividad y la violencia cobran especial relevancia y valor, también para algunas mujeres y, probablemente, como vía indirecta de afrontamiento de las dificultades existenciales. Entre los segundos cabe mencionar: la ausencia de apoyos familiares y sociales, que incrementa la vulnerabilidad de las mujeres; el efecto estresor resultante de la acumulación de dificultades en distintas esferas; el abuso de drogas por parte del varón, que puede actuar como detonante puntual; y la presencia de un entorno que, en ocasiones, tiende a no censurar el recurso a la violencia de género, propiciando una sensación de impunidad en los varones que facilita el recurso a la violencia.

Una diferenciación tan clara entre estos dos grupos de elementos, sin embargo, responde a necesidades analíticas, mientras que la realidad es infinitamente más compleja. Las interrelaciones entre los dos grupos de factores, de hecho, son estrechas y frecuentes, sea porque en la vida de las mujeres elementos de ambos grupos se funden y se confunden; sea porque un mismo elemento -como la presencia de circunstancias que "fuerzan" a las mujeres a iniciar una relaciónpuede incidir tanto en el proceso de formación de pareja como en las dinámicas de la relación.

No obstante, los resultados aportados pueden resultar de interés, por un lado, porque se ha tratado en todo momento de interpretar el papel de los factores de riesgo a la luz de las desigualdades estructurales subyacentes, evitando la "ceguera de género" que caracteriza mucha de la investigación en este ámbito (Ferrer y Bosch 2019); por otro, porque el análisis desarrollado profundiza en la interacción entre exclusión y violencia. Se trata de un acercamiento poco habitual, ya que la literatura en torno a la violencia de género no suele ocuparse de las situaciones de exclusión social en términos empíricos, a pesar de que varias investigadoras ya han subrayado la necesidad de examinar los efectos del impacto cumulativo de varios factores (White et al. 2011), algo que -como ya se ha apuntado- remite de forma muy directa a la noción de exclusión.

Los hallazgos del proceso analítico, además, amplían el abanico de elementos considerados por los estudios que se ocupan de factores de riesgo. Estos, de hecho, tienden a focalizar la mirada en los elementos que pueden favorecer la aparición de violencia de género en una pareja ya existente; mientras que casi ninguno analiza explícitamente los factores que inciden en el proceso de formación de pareja, abordado de manera contextualizada. En situaciones de exclusión, y teniendo en cuenta la rapidez con la que la violencia hace su aparición, parece lógico suponer que el peso de estos últimos es grande.

En relación con los factores que pueden facilitar la aparición de violencia de género en una pareja ya constituida, por otra parte, se ha intentado desarrollar un análisis complejo y contextualizado -que vaya más allá de la constatación habitual de factores estresores externos en ámbitos puntuales (desempleo, pobreza, falta de estudios, etc.)-. En este sentido, se ha tratado de avanzar en el análisis, 
examinando el impacto de varios elementos en la interacción y en el ejercicio de la violencia y, en lo que respecta más específicamente a los factores estresores, ahondando en las dinámicas de acumulación de dificultades en múltiples ámbitos (situación que, como ya se ha señalado, guarda una relación muy estrecha con procesos de exclusión).

El análisis realizado, por último, quizá pueda contribuir a la comprensión de mecanismos más generales de aparición y desarrollo de la violencia, al margen de la situación social de la mujer. Piénsese, por ejemplo, en cómo la presencia de elementos que fuerzan a las mujeres a encontrar una pareja puede fácilmente trasladarse a contextos sociales más normalizados: la razón de la premura o necesidad de encontrar pareja será seguramente diferente (muy probablemente no se tratará de resolver una situación de inseguridad residencial, sino de cumplir con el mandato social de género de formar una pareja y una familia o del miedo a la soledad, etc.), pero la consecuencia en términos de vulnerabilización de la posición subjetiva de algunas mujeres puede ir en la misma línea. O piénsese asimismo en el mecanismo de impunidad aprendida que se ha descrito en referencia a los hombres en exclusión social, pero que puede contribuir, más genéricamente, a desplazar la mirada analítica de las situaciones de violencia de las mujeres que la experimentan a los varones que la ejercen y a la propia relación, para un abordaje multicausal y multidimensional. Más concretamente, con tal formulación se ha pretendido subrayar que esta sensación de impunidad de los agresores -que no es innata sino aprendida poco a poco y con cada acto violento que no recibe sanción-facilita que paulatinamente se incremente la intensidad de la violencia que estos ejercen, en una espiral que no termina si no cesa la impunidad que la permite. Lo hemos descrito en relación a situaciones de exclusión, donde esta impunidad aparecía con gran claridad, pero puede aplicarse al conjunto de los procesos de violencia de género, contribuyendo a remarcar la importancia de la sensibilización y de la existencia de una sanción social y legal a los mismos.

\section{BIBLIOGRAFÍA}

Ahmad, F.; Riaz, S.; Barata, P. y Stewart, D. (2004): "Patriarchal beliefs and perceptions of abuse among South Asian immigrant women", Violence Against Women, 10(3), pp. 262-282. https://doi.org/10.1177/1077801203256000

Allman, D. (2013): The Sociology of Social Inclusion”, SAGE Open, 3(1), pp. 1-16. https://doi.org/10.1177/2158244012471957

Barrett, B.J.; Habibov, N. y Chernyak, E. (2012): "Factors Affecting Prevalence and Extent of Intimate Partner Violence in Ukraine: Evidence From a Nationally Representative Survey", Violence Against Women, 18(10), pp. 1147-1176. https://doi. org/10.1177/1077801212464387

Bonino Méndez, L. (2007): "Violencia de género y prevención. El problema de la violencia masculina”, en La violencia contra las mujeres. Prevención y detección, Madrid, Díaz de Santos, pp. 199-207. 
Boon, B. y Farnsworth, J. (2011): "Social Exclusion and Poverty: Translating Social Capital into Accessible Resources", Social Policy and Administration, 45(5), pp. 507-524. https://doi.org/10.1111/j.1467-9515.2011.00792.x

Chan, K.L. (2014): "Assessing the Risk of Intimate Partner Violence in the Chinese Population: The Chinese Risk Assessment Tool for Perpetrator (CRAT-P)", Violence Against Women, 20(5), pp. 500-516. https://doi. org/10.1177/1077801214535107

Choo, H.Y. y Ferree, M.M. (2010): "Practicing Intersectionality in Sociological Research: A Critical Analysis of Inclusions, Interactions, and Institutions in the Study of Inequalities", Sociological Theory, 28(2), pp. 129-149. https://doi. org/10.1111/j.1467-9558.2010.01370.x

Collins, P.H. (2000): Black Feminist Thought: Knowledge, Consciousness, and the Politics of Empowerment, Boston, Unwin Hyman.

Cunradi, C.B.; Ames, G.M. y Duke, M. (2011): “The relationship of alcohol problems to risk for unidirectional and bidirectional intimate partner violence among a sample of blue-collar couples", Violence and Victims, 26(2), pp. 147-158. https://doi. org/10.1891/0886-6708.26.2.147

Damant, D.; Lapierre, S. ; Kouraga, A. ; Fortin, A. ; Hamelin-Brabant, L. ; Lavergne, C. y Lessard, G. (2008): "Taking Child Abuse and Mothering Into Account", Journal of Women and Social Work, 23(2), pp. 123-133. https://doi. org/10.1177/0886109908314321

Damonti, P. (2017): Violencia de género en la pareja y exclusión social. Los efectos de la intersección entre diferentes estructuras de desigualdad (Tesis doctoral inédita). Departamento de Trabajo Social, Universidad Pública de Navarra, Pamplona, ES.

Damonti, P. (2019): "Exclusión social como factor de riesgo de violencia de género en la pareja”, Papers. Revista de Sociología, pp. 1-29. http://dx.doi.org/10.5565/rev/ papers. 2570

Elley, S. (2011): "Young Women, Class and gendered Heterosexuality: The Implication of Educational Aspirations and Social Networks for Sex Education Messages", Sociology, 45(3), pp. 413-429. https://doi.org/10.1177/0038038511399621

Ferrer-Pérez, V.A. y Bosch-Fiol, E. (2019): "El Género en el Análisis de la Violencia contra las Mujeres en la Pareja: de la "Ceguera" de Género a la Investigación Específica del Mismo”, Anuario de Psicología Jurídica, 29(1), pp. 69-76. https://doi. org/10.5093/apj2019a3

Flick, U. (2015): El diseño de Investigación Cualitativa, Madrid, Morata.

Flood, M. y Pease, B. (2009): "Factors influencing attitudes to violence against women", Trauma Violence Abuse, 10(2), pp. 125-142. https://doi. org/10.1177/1524838009334131

Fodor, E. (2006): "A Different Type of Gender Gap: How Women and Men Experience Poverty”, East European Politics and Societies, 20(1), pp. 14-39. https://doi. org/10.1177/0888325405284248

Franklin, C.A. y Menaker, T.A. (2014): “Feminism, Status Inconsistency, and Women's Intimate Partner Victimization in Heterosexual Relationships", Violence Against Women, 20(7), pp. 825-845. https://doi.org/10.1177/1077801214543385

Friedemann-Sánchez, G. y Lovatón, R. (2012): “Intimate Partner Violence in Colombia: Who Is at Risk?", Social Forces, 91(2), pp. 663-388. https://doi.org/10.1093/sf/ $\underline{\operatorname{sos} 131}$ 
Gabàs I Gasa, A. (2003): Indicadores de género contra la exclusión social. Informe sobre el fenómeno de la exclusión social, SURT, Asociació de Dones per a Inserció Laboral, disponible en http://www.surt.org/indicadors/es/docs/SURT.pdf [consulta 10-42020]

Gonzalez-Guarda R.M.; Vermeesch, A.L.; Florom-Smith, A.L.; Mccabe, B.E. y Peragallo, N.P. (2013): "Birthplace, Culture, Self-Esteem, and Intimate Partner Violence Among Community-Dwelling Hispanic Women”, Violence Against Women, 19(1), pp. 6-23. https://doi.org/10.1177/1077801212475336

Grose, R.G. y Grabe, S. (2014): “The Explanatory Role of Relationship Power and Control in Domestic Violence Against Women in Nicaragua: A Feminist Psychology Analysis", Violence Against Women, 20(8), pp. 972-993. https://doi. org/10.1177/1077801214546231

Hampton, R.; Oliver, W. y Magarian, L. (2003): "Domestic Violence in the African American Community", Violence Against Women, 9(5), pp. 533-577. https://doi. org/10.1177/1077801202250450

Illouz, E. (2012): Por qué duele el amor. Una explicación sociológica, Madrid, Clave intelectual Katz.

Jewkes, R. (2002): “Intimate partner violence: causes and prevention”, The Lancet, 359, pp. 1423-1429. https://doi.org/10.1016/S0140-6736(02)08357-5

Jewkes, R.; Levin, J. y Penn-Kekana, L. (2002): "Risk factors for domestic violence: findings from a South African cross-sectional survey", Social Science and Medicine, 55(9), pp. 1603-1617. https://doi.org/10.1016/S0277-9536(01)00294-5

Khalifeh, H.; Hargreaves, J.; Howard, L.M. y Birdthistle, I. (2013): "Intimate Partner Violence and Socioeconomic Deprivation in England: Findings From a National Cross-Sectional Survey", American Journal of Public Health, 103(3), pp. 462-472. https://doi.org/10.2105/AJPH.2012.300723

Kiss, L.; Schraiber, L.B.; Heise, L.; Zimmerman, C.; Gouveia, N. y Watts, C. (2012): "Gender-based violence and socioeconomic inequalities: Does living in a more deprived neighborhoods increase women's risk of intimate partner violence?", Social Science and Medicine, 74(8), pp. 1172-1179. https://doi.org/10.1016/j.socscimed.2011.11.033

Kuckartz, U. (2014): Qualitative Text analysis: A Guide to Methods, Practice and Using Software, Londres, Sage. https://doi.org/10.4135/9781446288719

Kvale, S. (2011): Las entrevistas en Investigación Cualitativa, Madrid, Morata.

Lagarde, M. (2008): "Amor y sexualidad, una mirada feminista", en Curso de verano: Sexualidades en movimiento, derechos a debate, Santander, UIMP-Universidad Internacional Menéndez Pelayo.

Lanier, C. y Maume, M. (2009): "Intimate Partner Violence and Social Isolation Acros the Rural/Urban Divide", Violence Against Women, 15(11), pp. 1311-1330. https:// doi.org/10.1177/1077801209346711

Laparra, M.; Obradors, A.; Pérez, B.; Pérez, M.; Renes, V.; Sarasa, S.; Subirats. J. y Trujillo, M. (2007): "Una propuesta de consenso sobre el concepto de exclusión. Implicaciones metodológicas", Revista Española Del Tercer Sector, 5, pp. 15-57.

Mavrikiou, P.M.; Apostolidou, M. y Parlalis, S.K. (2014): "Risk factors for the prevalence of domestic violence against women in Cyprus", The Social Science Journal, 51(2), pp. 295-301. https://doi.org/10.1016/j.soscij.2014.02.002 
Nóblega-Mayorga, M. (2012): "Risk and Protective Factors for Physical and Emotional Intimate Partner Violence Against Women in a Community of Lima, Peru", Journal of Interpersonal Violence, 27(18), pp. 3644-3659. https://doi. org/10.1177/0886260512447522

Panchanadeswaran, S.; Ting, L.; Burke, J.G.; O'campo, P.; Mcdonnel, K. y Gielen, A.C. (2010): "Profiling Abusive Men Based on Women's Self-Reports: Finding From a Sample of Urban Low-Income Minority Women", Violence Against Women, 16(3), pp. 313-327. https://doi.org/10.1177/1077801209360854

Peralta, R.L.; Tuttle, L.A. y Steele, J. (2010): “At the Intersection of Interpersonal Violence, Masculinity, and Alcohol Use: The Experiences of Heterosexual Male Perpetrators of Intimate Partner Violence", Violence Against Women, 16(4), pp. 387-409. https://doi.org/10.1177/1077801210363539

Raj, A. y Silverman, J. (2002): "Violence Against Immigrant Women", Violence Against Women, 8(3), pp. 367-398. https://doi.org/10.1177/10778010222183107

Renner, L.M. y Whitney, S.D. (2012): "Risk factors for unidirectional and bidirectional intimate partner violence among young adults", Child Abuse and Neglect, 36(1), pp. 40-52. https://doi.org/10.1016/j.chiabu.2011.07.007

Ruiz-Jarabo, C. y Blanco Prieto, P. (2007): La violencia contra las mujeres. Prevención y detección, Madrid, Díaz de Santos.

Saraví, G.A. y Makowski, S. (2011): "Social Exclusion and Subjectivity: Youth Expressions in Latin America", The Journal of Latin American and Caribbean Anthropology, 16(2), pp. 315-334. https://doi.org/10.1111/j.1935-4940.2011.01160.x

Seligman, M.E.P. (1975): Helplessness. On depression, Development, and Death, San Francisco, W.H. Freeman \& Company.

Shaaban, S. (2011): "A Conceptual Framework Review of Social Exclusion and its Relations with Social Cohesion and Poverty in Europe", The International Journal of Diversity in Organization, Communities and Nations, 11(1), pp. 117-131. https://doi. org/10.18848/1447-9532/CGP/v11i01/38952

Sokoloff, N.J. (2004): "Domestic Violence at the Crossroads. Violence Against Poor Women and Women of Color", Women's Study Quarterly, 32(3/4), pp. 139-147.

Sokoloff, N.J. y Dupont, I. (2005): "Domestic Violence at the Intersections of Race, Class and Gender", Violence Against Women, 11(1), pp. 38-64. https://doi. org/10.1177/1077801204271476

Stöckl, H.; Heise, L. y Watts, C. (2011): "Factors associated with violence by a current partner in a nationally representative sample of German women", Sociology of Health and Illness, 33(5), pp. 694-709. https://doi. org/10.1111/j.1467-9566.2011.01319.x

Strier, R.; Sigad, L.; Eisikovitz, Z. y Buchbinder, E. (2014): "Masculinity, Poverty and Work: The Multiple Constructions of Work among Working Poor Men”, Journal of Social Policy, 43(2), pp. 331-349. https://doi.org/10.1017/S0047279413000949

Subirats, J. (dir.) (2004): Pobreza y exclusión social. Un análisis de la realidad española y europea, Barcelona, Fundación "la Caixa".

Subirats, J. (dir.) (2005): Análisis de los factores de exclusión social, Bilbao, Fundación BBVA.

Van Bergen, A.; Hoff, S.; Van Ameijden, E. y Van Hemer, A. (2014): “Measuring Social Exclusion in Routine Public Health Surveys: Construction of a Multidimensional Instrument", Plos One, 9(5), pp. 1-11. https://doi.org/10.1371/journal.pone.0098680 
Van Vijk N. Ph. L. y De Brujin, J.G.M. (2012): "Risk Factors for Domestic Violence in Curacao", Journal of Interpersonal Violence, 27(15), pp. 3032-3053. https://doi. org/10.1177/0886260512441074

Vives-Cases, C.; Torrubiano-Domínguez, J.; Escribà-Agüir, V.; Ruiz-Pérez, I.; Montero-Piñar, M.I. y Gil-González, D. (2011): "Social Determinants and Health Effects of Low and High Severity Intimate Partner Violence", Annals of Epidemiology, 21(12), pp. 907-13. https://doi.org/10.1016/j.annepidem.2011.02.003

Walker, L.E. (1984): The Battered Woman Syndrome, Nueva York, Springer Publishing Company.

White, J.W.; Koss, M.P. y Kazdin, A.E. (2011): Violence against women and children. Mapping the terrain. Vol. 1, Washington, American Psychological Association. https://doi.org/10.1037/12307-000

Yick, A. (2001): "Feminist Theory and Status Inconsistency Theory", Violence Against Women, 7(5), pp. 545-562. https://doi.org/10.1177/10778010122182596 